\title{
Guiding principles for a successful multidisciplinary research collaboration
}

\section{Future Science OA

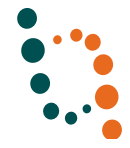

\author{
"The value of a collaborative project extends to training highly qualified \\ personnel and expanding the breadth of knowledge of even the most seasoned \\ expert involved...
}

Keywords: $\bullet$ grant funding $\bullet$ guidelines $\bullet$ mentorship $\bullet$ multidisciplinary collaboration
$\bullet$ productivity

Solving the most critically important scientific problems or engineering groundbreaking technologies often requires teams of researchers from different backgrounds to work together. One might need to make new materials (chemistry), characterize and understand the properties of the material (physics), assemble the material into a useful device (engineering), address the biological problem to be solved (biology) and demonstrate utility in patients (medicine). A single laboratory is unlikely to have all of the expertise necessary to address complex problems that can make a significant impact on society.

The merging of fields also makes otherwise impossible goals achievable, often in a timelier manner. This has led many granting agencies, academic and commercial institutions to encourage the development of interdisciplinary teams $[1-3]$. As a result, there has been an increase in the quantity and quality of publications combining the work of authors with diverse backgrounds. In the collaborative setting, trainees and principal investigators (PIs) alike become immersed in different areas of study, research styles and how fields beyond their own familiar worlds operate. These aspects introduce challenges to ensuring a team is productive and moving toward their research objectives. However, when the team is working well, this can be a particularly useful learning experience, leading to exceptionally well-rounded trainees that will learn from a variety of experts. While the idea of assembling the best-of-the-best scientists and engineers to address an important problem in society is of merit, the practical aspects of working together can be challenging. Ensuring success hinges on effective communication - knowing what and how best to convey thoughts and opinions.

Our three research laboratories have different research experience, interests and backgrounds: we have merged together on a project that involves the identification, delivery and assessment of small peptide cancer drugs. Over the last 4 years, we have worked together to characterize and evaluate these drugs through shared funding from the Collaborative Health Research Program in Canada. Through this joint project, we have learned to be effective collaborators with one another, started to publish our results [4], and through the years, we have shared with one another experiences of good and bad collaborations. While there are a number of excellent commentaries to describe how teams in research work together $[5,6]$, we thought an article that specifically focuses on practical tips that are important in building strong team dynamics and ensuring that the multidisciplinary research project is conducted in an efficient and productive manner in an academic setting would be useful for guiding other academic collaborators. Additionally, some of these principles may be further extended to include networking and academic-industrial collaborations.

\section{Learn the language}

One of the biggest challenges in working together is building a communication strategy that is aligned with all researchers. Each research discipline, and often each labora-

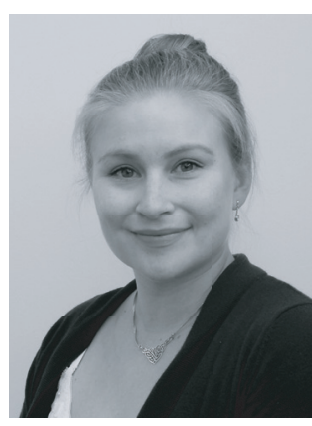

Lindsay C Lustig ${ }^{1,2}$, Romina Ponzielli', Peter S Tang ${ }^{3}$, Sarmitha Sathiamoorthy ${ }^{4}$, Ichiro Inamoto ${ }^{4}$, Jumi A Shin ${ }^{4}$, Linda Z Penn ${ }^{1,2}$ \& Warren CW Chan ${ }^{\star 3,5}$

'Princess Margaret Cancer Centre, University Health Network, 101 College Street, Toronto, ON, M5G 1L7, Canada ${ }^{2}$ Department of Medical Biophysics, University of Toronto, 101 College Street, Toronto, ON, M5G 1L7, Canada ${ }^{3}$ Institute of Biomaterials \& Biomedical Engineering, Donnelly Centre for Cellular \& Biomolecular Research, University of Toronto, 160 College St., Toronto, ON, M5S 3G9, Canada

${ }^{4}$ Department of Chemistry, University of Toronto, 3359 Mississauga Road, Mississauga, ON, L5L 1C6, Canada ${ }^{5}$ Department of Chemistry, Chemical Engineering, Materials Science \& Engineering, University of Toronto, 160 College St., Toronto, ON, M5S 3G9, Canada

*Author for correspondence: warren.chan@utoronto.ca

FUTURE SCIENCE partof 
tory, has unique 'language'. Occasionally, the same terms can be defined completely differently depending on the discipline or even from one research group to another. Commonly used jargon and terms should be clearly defined and collaborators that are relatively new to your field may require more thorough explanations. So, patience in explaining concepts is required.

\section{Address differences in operation}

The way laboratories operate can differ significantly. For example, biologists, chemists and physicists tend to use the principles of 'hypothesis' to drive research projects, but many engineers do not use this research model. To address these differences, a simple communication strategy should be developed at the beginning of the collaboration. All PIs should discuss and develop useful shared reporting tools to bridge the differences between the researchers. An example is a monthly report with objectives, procedures, experimental results and challenges. These reports can be circulated before monthly meetings. Communication is the key to developing a productive team.

\section{Make an operational plan}

Solidify critical discussions by creating a policy document for the collaboration at the start of the project. Each member should sign off on this agreement. If a document is not created, researchers tend to forget the agreed-upon collaboration principles, which can potentially lead to problems during the course of the project. Some of the points of discussion that should be included in a policy document are:

- What is the role of each party on the project and what are the objectives of each party in accomplishing the goals of the project?

- How will each party train its researchers on the project?

- How can the project be communicated between the different research groups?

- What strategies will be used to address problems with the project and personnel?

- What are the privacy issues for each party? Can the researchers discuss the project with other members of their own research laboratories? If there are patents created, how will they be reported?

- What are the different timelines of each group's contribution to the team effort, and how will milestones and expectations be managed?
Outlining the principles of engagement and reporting can form the groundwork for productive communication and discovery of novel advances.

\section{Share the credit}

Develop a system to provide appropriate credit to all researchers who participate in the project. If the outcome of the study leads to a paper, how will authorship be organized? Who will be responsible for writing the manuscript $(s)$, and what will be their emphasis? If there are patents created, who is included on the patent? Some of us have experienced a PI who wants all of the credit but does very little work [7]. The allocation of credit should be discussed early among all the contributors. This discussion should extend to trainees in addition to PIs and will often be initiated by the latter. Much overlap exists with respect to the reasons the PIs and trainees are involved in a given research collaboration. For instance, both PIs and trainees are united by the common drive to disseminate high-impact, quality data, but there are usually additional interests that may differ between the parties involved, such as a PI who is preparing his or her promotion package and needs to publish papers in certain types of journals. If these are considered, it will likely improve the collaborative experience for all, and importantly, increase productivity and impact.

Consider the trainees involved in conducting the work. Authorship is of great importance to them as well, for instance, for completing a degree or building one's curriculum vitae. Collaborators should discuss who will be credited with authorship and the order of names appearing on the different published works that arise as a result of combined efforts. Although this can be an uncomfortable topic and is undoubtedly a challenge to decide early in the process, ensuring constant and consistent communication on where individuals stand on authorship, particularly as the projects evolve, avoids unnecessary worry and eliminates misguided expectations on all parts. Remember, as with any endeavor, if individuals know the reward, they will adjust their expectations and be more motivated to do the work.

\section{Share the money}

Once a team has successfully obtained funding for a project, it is important from the onset that all PIs agree to share the financial resources. There are cases where the lead PI decides to control all the funding rather than share it from the onset and will try to use the money to control the authorship and project objectives. Such behavior can sabotage a good collaborative project. Currently, most granting agencies do not have a checks-and-balance system, and this behavior 
can propagate. All parties should develop a financial plan that is beneficial to the project as a whole such that the lead investigator cannot and should not use finance as a way to manipulate authorship on patents, talks and papers. A budget plan should be written to indicate which laboratory does what and how much money is required to get each part of the project done. Everybody should learn to be reasonable as the funding is not bottomless.

\section{Discuss project plans \& time management}

At the beginning of any collaboration, it is important to develop a project plan. Researchers should indicate the objectives of each facet of the project, the long- and shortterm goals, procedures and activities, as well as who will be needed to perform each of the activities. Each project should have tangible milestones and deliverables that are clearly identified and written. This plan will identify each researcher's activities, allow subprojects to be evaluated during the course of the bigger project, and allow the team to modify goals as the project evolves over the course of the experiments. It also removes any ambiguity regarding who did the work. Related to this is the importance of understanding the amount of time that is needed to complete different experiments. Depending on the field of study or the specific experiment, more or less time might be required to achieve results than researchers outside of the field would expect. Taking the time to explain what is involved in performing each task helps to keep everyone on the same page and encourages realistic expectations.

\section{"While the idea of assembling the best-of-the-best scientists and engineers to address an important problem in society is of merit, the practical aspects of working together can be challenging."}

This is also important when assessing, for instance, the appropriate times for trainees to complete their graduate degrees or postdoctoral studies. In many collaborations, the work completed by one individual relies on the results of another. To avoid situations where the progress of one trainee's course of study is stalled due to the completion of anothers' task, any potential setbacks should be considered in advance with feasible timelines devised and backup plans in place. It may be wise for a trainee to work concurrently on an additional, unrelated project in case the collaborative project does not go as expected.

\section{Hold frequent meetings}

It is highly recommended for groups to hold routine meetings, for example, once a month. It is useful for each laboratory to submit a short activity report electronically for all team members to view beforehand. These activity reports allow the team to follow the progress of each project, evaluate the data and offer suggestions to help plan downstream experiments. Having more eyes and ears present, particularly from different disciplines, can bring to the forefront various assumptions that are being made in planning experiments, and stimulate conversations regarding how best to establish criteria for moving forward or interpreting datasets. Regrouping on a regular basis serves as a continuous reminder of the team's end goal beyond an individual experiment, because several persons are involved to offer input and help maintain focus. This in turn translates to greater research productivity. Activity reports also provide a regular platform for trainees to present their work and, in doing so, accelerates their ability to effectively communicate their research to persons of different scientific backgrounds and levels of understanding. The monthly meetings can also be an opportunity for trainees to participate in discussions regarding strategies for drafting grant applications, and the planning and considerations therein. As an integral part of the research process, it can be of great benefit for the trainees to actively participate in the planning and execution of securing funding. In addition, holding one or two meetings per year in person reinforces the connections between the team. This familiarity will continue to be informally driven by trainees, as they feel comfortable picking up the phone and calling teammates in the other laboratories.

\section{Encourage open communication: be fair \& respectful}

It is very important that every aspect of the project be dealt with fairly. A failed collaboration often results from a researcher not being given proper credit or enough money to do the project. Should one of the collaborative members feel that there is inequality in the project, it must be addressed immediately. As mentioned, we suggest that the partitioning of funding and assignment of credit be discussed early and ideally, be mapped to the project plan. It is imperative for everyone to be open and honest within the team, and it is equally important for this to be done tactfully. For instance, if a project is not working, the researchers should discuss it. If there are issues with funding or problems with the personnel, the PIs should discuss it. This may seem obvious, but often team members choose not to initiate these critical conversations. When certain issues that are sensitive arise, it is important for the PIs to come together and talk privately prior to discussing their decisions with the team. The same should be said about the trainees who are on the 
project. Lastly, the relationship between a PI and his or her trainee who is participating in a collaboration must always be respected. Working alongside several PIs can provide an abundance of invaluable opportunities to learn and enhance the trainee experience. However, it also means managing the needs of more than one supervisor, and unfortunately the potential for conflict should another PI overstep his/her authority. Such issues can be avoided if all parties involved appreciate that although group discussion with respect to a trainee's direction in a project is imperative, it is ultimately the trainee along with the PI who make the final decision. Having a PI directly demand tasks from another PI's personnel will inevitably create strife that could easily be avoided.

\section{Conclusion}

The benefits of collaboration are vast $[3,6]$. Some will be obvious from the onset of the project, while others may go unrealized until months and years later. In a collaboration, the researchers should work in synergy, complementing one another to complete the essential toolset necessary to achieve the end goal. The merging of diverse fields can yield breakthroughs in a speed unachievable if those contributors were to work independently. The value of a collaborative project extends to training highly qualified personnel

\section{References}

1 Adams J. The rise of research networks. Nature 490 (7420), 335-336 (2012).

2 Carpenter JF. Value that industrial collaborations bring to research and education efforts in universities: perspective of a professor working in the field of development of therapeutic proteins. Hum. Genomics 7(7), 1-3 (2013).

3 Dozier AM, Martina CA, O’Dell NL et al. Identifying emerging research collaborations and networks: method development. Eval. Health Prof. 37(1), 19-32 (2014). and expanding the breadth of knowledge of even the most seasoned expert involved, which can spill over and enhance other research endeavors underway or in the future. Like many undertakings with great potential, collaboration in science can be one of high risk in addition to high reward. Effective team management and open communication from the very first discussion will maximize the likelihood of success and productivity.

\section{Financial \& competing interests disclosure}

The authors thank the Canadian Institute of Health Research, Natural Sciences Engineering Research Council of Canada, Canadian Research Chairs Program, and Collaborative Health Research Program for financial support of their research activities. The authors have no other relevant affiliations or financial involvement with any organization or entity with a financial interest in or financial conflict with the subject matter or materials discussed in the manuscript apart from those disclosed.

No writing assistance was utilized in the production of this manuscript.

\section{Open Access}

This work is licensed under the Creative Commons Attribution 4.0 License. To view a copy of this license, visit http://creativecommons.org/licenses/by/4.0/

4 Tang PS, Sathiamoorthy S, Lustig LC et al. The role of ligand density and size in mediating quantum dot nuclear transport. Small 10(20), 4182-4192 (2014).

5 Vincens Q, Bourne PE. Ten simple rules for a successful collaboration. PLoS Comput. Biol. 3(3), e44 (2007).

6 Hanawalt PC. Research collaborations: trial, trust, and truth. Cell 126(5), 823-825 (2006).

7 Abu-Zaid A, Alnajjar A, Anwer LA. Authorship disintegrity in research collaborations: ends do not justify means in science. Med. Educ. Online 19, 24930 (2014). 